\title{
The functional and structural characteristics of the emotion network in alexithymia
}

This article was published in the following Dove Press journal:

Neuropsychiatric Disease and Treatment

\author{
Dai Han ${ }^{1-3}$ \\ Mei $\mathrm{Li}^{4}$ \\ Minjun $\mathrm{Mei}^{4}$ \\ Xiaofei Sun ${ }^{4}$ \\ 'Institutes of Psychological Sciences, \\ Hangzhou Normal University, \\ Hangzhou, Zhejiang, China; ${ }^{2}$ Children \\ and Adolescents Mental Health \\ Joint Clinic, The Affiliated Hospital \\ of Hangzhou Normal University, \\ Hangzhou, Zhejiang, China; ${ }^{3}$ Zhejiang \\ Key Laboratory for Research in \\ Assessment of Cognitive Impairments, \\ Hangzhou, Zhejiang, China; ${ }^{4}$ Mental \\ Health Education and Counseling \\ Center, Hangzhou Normal University, \\ Hangzhou, Zhejiang, China
}

Background: Alexithymia is a multifaceted personality trait characterized by emotional dysfunction.

Methods: In this study, the functional and structural features of the emotion network in alexithymia were investigated using resting-state functional MRI (rsfMRI), voxel-based morphometry (VBM), functional connectivity (FC) analysis, and diffusion tensor imaging (DTI). Alexithymic and non-alexithymic students were recruited from the local university. The intrinsic neural activity and gray matter density of the brain regions in the emotion network were measured using rsfMRI and VBM; the FC and structural connectivity of the brain regions in the emotion network were measured using FC analysis and DTI.

Results: The altered intrinsic neural activity in V1, rostral dorsal anterior cingulate cortex, and left amygdala, and the weak FC between V1 and left superior temporal gyrus and V1 and left paracentral lobule in alexithymia subjects were identified. However, no alteration of the structure and structural connectivity of the emotion network was identified.

Conclusion: The results indicated that the development of alexithymia might have been caused only by slight alteration of the neural activity. Furthermore, the results suggest that noninvasive treatment technologies for improving the brain activity are suitable for alexithymic individuals. Keywords: emotion network, rsfMRI, VBM, functional connectivity, structural connectivity

\section{Introduction}

Alexithymia is a personality trait characterized by difficulties in identifying and describing one's own emotional feelings and an external thinking style. ${ }^{1}$ Alexithymia is associated with increased risks for psychosomatic complaints, autism, anxiety, and depression disorder. ${ }^{2-5}$ Moreover, impaired identification and expression of emotions (the core symptoms of alexithymia) may play a mediating role in these disorders. ${ }^{2-4,6,7}$ Clarifying the neural mechanism of emotional dysfunction in alexithymic individuals can improve the understanding of this personality trait and its relationships with psychosomatic disorders.

The neurobiological basis of the emotion function has been a research focus for a long time. Through a meta-analysis study, an emotion network covering 21 brain areas was identified (Table 1). ${ }^{8}$ Notably, the distinct structural or functional abnormalities of the brain regions in the emotion network have been linked to the specific emotional symptoms of different mental disorders. ${ }^{9}$ For instance, in the case of schizophrenic individuals, unusual activities are seen in the amygdala, insula, and ventral striatum in response to emotional stimulus, and a reduced gray matter (GM) volume is observed in the frontal cortex..$^{10,11}$ In the case of depressed patients, abnormal activities are seen in the amygdala, anterior insula, ventral striatum, and prefrontal cortex (PFC) in response to emotional stimuli, and reduced volume is observed in the amygdala,
Correspondence: Dai Han Institutes of Psychological Science, Hangzhou Normal University, 2318 Yuhangtang Road, Hangzhou, Zhejiang, 3III2I, China

Email handai@hznu.edu.cn 
Table I The MNI coordinates of the selected ROls in the emotion network

\begin{tabular}{|c|c|c|c|c|}
\hline Name & $\mathbf{x}$ & $\mathbf{Y}$ & $\mathbf{Z}$ & $\begin{array}{l}\text { Radius } \\
(\mathrm{mm})\end{array}$ \\
\hline Visual area 8/bilateral superior cerebellum (V8/sCB, Bi) & -11 & -62 & -20 & 12 \\
\hline Bilateral middle temporal complex $(\mathrm{MT}+, \mathrm{Bi})$ & -28 & -69 & 9 & 12 \\
\hline Right visual area $4(\mathrm{~V} 4, \mathrm{R})$ & 44 & -79 & -4 & 12 \\
\hline Right lateral occipital cortex/temporal lobe (latOCC/Temp, R) & 49 & -56 & -2 & 12 \\
\hline PCC & I & -54 & 25 & 12 \\
\hline Primary visual cortex (VI) & -7 & -89 & 0 & 12 \\
\hline Dorsal medial prefrontal cortex (dmPFC) & -2 & 51 & 29 & 12 \\
\hline Pregenual anterior cingulate cortex ( $\mathrm{PgACC})$ & I & 39 & 2 & 12 \\
\hline Rostral dorsal anterior cingulate cortex (rdACC) & 0 & 29 & 25 & 12 \\
\hline Ventral anterior insula/frontal operculum/right temporal pole, bilateral posterior & 38 & 19 & -11 & 12 \\
\hline \multicolumn{5}{|l|}{ Orbitofrontal cortex (valns/frOP/TP, RpOFC, Bi) } \\
\hline Anterior insula, posterior orbitofrontal cortex (alns, pOFC, Bi), & -40 & 23 & -6 & 12 \\
\hline Bilateral ventral anterior insula/temporal cortex/orbitofrontal cortex (valns/TC/OFC, Bi) & -7 & 12 & -23 & 12 \\
\hline Bilateral middle insula, right dorsal putamen (midlns, Bi dPut, R) & 21 & 0 & 2 & 12 \\
\hline Left ventral Striatum, middle insula, hippocampus (vStr, midlns, HCMP, L) & -36 & 0 & -8 & 12 \\
\hline Left Basolateral Amygdala (Amy_L) & -23 & -3 & -21 & 12 \\
\hline Hypothalamus $(\mathrm{Hy})$ & -7 & -5 & -13 & 12 \\
\hline Periaqueductal gray/thalamus (PAG/Thal) & -1 & -23 & 2 & 12 \\
\hline Bilateral amygdala, ventral striatum, ventral globus pallidus, thalamus (Amy vStr vGP Thal, Bi) & 11 & -6 & -10 & 12 \\
\hline Right frontal operculum (frOP, R) & 46 & 24 & 6 & 12 \\
\hline Right inferior frontal gyrus (IFG, R) & 49 & 20 & 19 & 12 \\
\hline Left pre-supplementary motor area, inferior frontal gyrus (preSMA, IFG, L) & -23 & 13 & 37 & 12 \\
\hline
\end{tabular}

Abbreviations: MNI, Montreal Neurological Institute; PCC, posterior cingulate cortex; ROI, region of interest.

ventral striatum, anterior cingulate gyrus (ACC), PFC, and hippocampus. ${ }^{12-14}$ Moreover, abnormal neural activity in the amygdala, insula, ACC, medial PFC, and posterior cingulate cortex (PCC) is observed in alexithymic individuals. ${ }^{15}$ Two voxel-based morphometry (VBM) studies revealed decreased GM density in the ACC and left amygdala and increased GM density in the right posterior insula and right cingulate cortex in alexithymic individuals. ${ }^{16,17}$

It is notable that alexithymia is related to abnormal activity in multiple brain regions instead of only a single region. Multiple brain regions have to cooperate with each other to maintain the brain functions. Therefore, it is reasonable to hypothesize that the occurrence of alexithymia is associated with not only functional or structural abnormalities of some brain regions in the emotion network but also some abnormal functional connectivity (FC) or structural connectivity among these brain regions. Resting-state functional MRI (rsfMRI) analyses on FC provide a powerful method to investigate the FC among brain regions. Liemburg found abnormal FC levels among several brain regions (such as the medial PFC, occipital areas, insula cortex, and ACC) in alexithymic individuals. ${ }^{18}$ Diffusion tensor imaging (DTI) is a highly sensitive method for measuring the structural integrality of the white matter tracts that connect brain regions. ${ }^{19}$ Structural connectivity is also an important index to investigate the cooperative effects among the brain regions in the emotion network. A DTI study conducted by Ho et al suggested that the structural connectivity of the superior longitudinal fasciculus was significantly correlated with alexithymia. ${ }^{20}$ These studies partly supported the hypothesis presented earlier. Unfortunately, Liemburg's study investigated only the brain regions in the default network. Ho et al focused only on the corpus callosum and superior longitudinal fasciculus. In order to verify the hypothesis proposed earlier, it is necessary to investigate the functional and structural features of the emotion network in alexithymic individuals.

In order to investigate the structural and functional features of the emotion network in alexithymia, the present study included four programs: first, examining the neural intrinsic activity of the brain regions in the emotion network using rsfMRI; second, examining the structural features of these brain regions using VBM analysis; third, examining the FCs among the brain regions showing abnormal neural intrinsic activity or abnormal structural features in the emotion network via FC analysis; and fourth, examining the structural connections among the brain regions showing abnormal FC in the emotion network via DTI analysis. Holistic knowledge on the emotion network will contribute to a deeper understanding of the neural nature of alexithymia. 


\section{Methods}

\section{Subjects}

All the participants were recruited from among the freshmen students of a local university. All the students underwent a mental health checkup (including alexithymia measurement, see the "Psychological measurements" section) during the university entrance examination in 2015. The participants satisfied the following inclusive criteria: right-handed and physically healthy individuals without histories of traumatic brain injuries, substance abuse, or mental disorders. Participants were excluded if they had contraindications to MRI scans or had consumed any psychotropic medications within 6 months before the study. The eligible participants with alexithymia were randomly chosen, telephonically contacted, and assigned to the alexithymia group (Alex group). The eligible non-alexithymia participants, who matched the alexithymia subjects with regard to age and sex, were randomly chosen, contacted by telephone, and assigned to the healthy control group (HC group). All the protocols were approved by the Research Ethics Review Board of Hangzhou Normal University. Thus, 42 participants (21 each in the Alex and HC groups) were recruited in the present study. The participants were informed of the study protocol and signed the informed consent form.

\section{Psychological measurements}

The characteristics of alexithymia were evaluated by the Toronto Alexithymia Scale-20 (TAS-20), which is a 20-item self-report scale, rating from 1 (strongly disagree) to 5 (strongly agree). The participants with TAS-20 total scores $>60$ and $<52$ were assigned to Alex group and $\mathrm{HC}$ group, respectively. ${ }^{21}$

\section{MRI measurements}

All MRI scan processes were performed with a 3T wholebody MR imager (GE MR750). The rsfMRI scans were acquired: sequence $=$ gradient recalled echo-echo planar images, axial slices, 43 slices, repetition time (TR)/echo time $(\mathrm{TE})=2,000 / 30 \mathrm{~ms}$, field of view $(\mathrm{FOV})=220 \times 220 \mathrm{~mm}^{2}$, resolution $=64 \times 64$, flip angle $(\mathrm{FA})=90^{\circ}$, total scan time $=480 \mathrm{~s}$. Participants were instructed to simply rest with their eyes closed, not to think of anything in particular, and not to fall asleep. The 3D T1-weighted images were acquired: sagittal slices, 180 slices, $\mathrm{TR} / \mathrm{TE}=8,100 / 3 \mathrm{~ms}$, resolution $=256 \times 256$, $\mathrm{FOV}=256 \times 256 \mathrm{~mm}^{2}$, thickness $/ \mathrm{gap}=1 / 0 \mathrm{~mm}, \mathrm{FA}=8^{\circ}$, total scan time $=305 \mathrm{~s}$. Lastly, DTI was acquired: spin-echo echoplanar imaging sequence, 67 axial slices, TR/TE $=8,600$ / minimum $\mathrm{ms}, \mathrm{FOV}=192 \times 192 \mathrm{~mm}^{2}$, matrix $=128 \times 128$, number of shots $=1$, slice thickness $=1.5 \mathrm{~mm}$. Diffusion direction: TENSOR, 30 directions, $b$-value $=1,000 \mathrm{~s} / \mathrm{mm}^{2}$,
NEX (number of excitations) $=2$, number of $\mathrm{T} 2$ images $=4$, voxel size $=1.5 \times 1.5 \times 1.5 \mathrm{~mm}^{3}$, total scan time $=593 \mathrm{~s}$.

\section{Data processing}

rsfMRI data analysis and statistical analysis

Based on the MatLab software, data preprocessing was performed by the Data Processing Assistant for Resting-State fMRI (DPARSF, http://www.restfmri.net) and the rsfMRI data analysis toolkit (REST, http://www.restfmri.net). ${ }^{22,23}$ The images were corrected for slice-time differences, realigned for head motion correction, co-registered to T1, and spatially smoothed, according to a previous study's suggestion. ${ }^{24}$ The amplitude of low-frequency fluctuation (ALFF) map was calculated and the ALFF values of 21 regions of interest (ROI) in the emotion network were extracted. The ROI selection was based on the previous studies (Table 1). ${ }^{8,25}$ The ALFF values of the two groups were compared using a independent $t$-test by SPSS19.0. Differences were considered significant at a two-sided $p$-value of 0.05 .

\section{VBM data analysis and statistical analysis}

Data were processed using the VBM toolbox (VBM8, http:// dbm.neuro.uni-jena.de/vbm.html) in a statistical software package (SPM8, http://www.fil.ion.ucl.ac.uk/spm/). Then preprocessing was undertaken: the $\mathrm{T} 1$-weighted images were bias-corrected and segmented into GM, white matter (WM), and cerebrospinal fluid (CSF); Diffeomorphic Anatomical Registration Through Exponentiated Lie Algebra (DARTEL) create template; DARTEL existing template; normalize to Montreal Neurological Institute space; spatial smoothing with $8 \mathrm{~mm}$ full-width at half-maximum Gaussian kernel; the GM density of 21 ROIs in the emotion network was extracted. The GM density of the two groups was compared using independent $t$-test by SPSS19.0. Differences were considered significant at two-sided $p$-value of 0.05 .

\section{Functional connectivity analysis and statistical analysis}

The FC between each ROI in the emotion network and other brain regions in the whole brain was determined using REST software. According to the previous studies, the preprocessing was undertaken: time series extraction, Pearson's correlation analysis of ROIs and whole-brain voxel time series (WM, CSF, and six head motion parameters were covariates), Fisher's Z-transformation, voxel-by-voxel based comparisons of FC of 21 ROIs between two groups by two-sample $t$-test using REST, and AlphaSim correction $(p<0.001$, AlphaSim correction, cluster size $>20) .{ }^{26,27}$ The result was visualized by BrainNet Viewer (https://www.nitrc.org/projects/bnv/). ${ }^{28}$ 
Table 2 Comparison of the demographic variables and psychological measurements between the Alex and the $\mathrm{HC}$ groups

\begin{tabular}{|c|c|c|c|}
\hline Variables & $\begin{array}{l}\text { Alex group, } \\
n=2 \text { I }\end{array}$ & $\begin{array}{l}\text { HC group, } \\
n=2 I\end{array}$ & $p$-value \\
\hline \multicolumn{4}{|l|}{ Demographic data } \\
\hline Males, n (\%) & II (52.4) & II (52.4) & \\
\hline Age (years) & I8.33 (1.06) & $18.62(1.07)$ & $p=0.39$ \\
\hline TAS-20 scores* & $58.10(3.2)$ & $41.00(7.75)$ & $p<0.001$ \\
\hline
\end{tabular}

Notes: Data are presented as percentage/ratio or mean $\pm S D ; *_{p}<0.05$.

Abbreviations: Alex, alexithymia; HC, healthy control; TAS-20, Toronto Alexithymia Scale-20.

Structural connectivity analysis and statistical analysis The DTI data were processed using the Pipeline for Analyzing Brain Diffusion Images (PANDA, http://www. nitrc.org/projects/panda/) and DTI Studio (https://www. mristudio.org/). The DTI data procession included transferring Digital Imaging and Communications in Medicine to Neuroimaging Informatics Technology Initiative, image registration, normalizing, and smoothing. Maps of FA were computed from the DTI data. Based on the FC analysis results in the present study, the FA values were extracted using the Fiber Assignment by Continuous Tracking (FACT) algorithm. ${ }^{29}$ The FA values of the two groups were compared using independent $t$-test by SPSS.
Differences were considered significant at two-sided $p$-value of 0.05 . The result was visualized by BrainNet Viewer.

\section{Results \\ Demographic characteristics and psychological measurements of the students} The results indicated no significant difference in the demographic variables between the two groups. TAS-20's global and three subscales' scores in the Alex group were significantly higher than those in the HC group (Table 2).

\section{Comparison analysis of rsfMRI between two groups}

The comparative analysis revealed significant differences in ALFF values of V1, rostral dorsal anterior cingulate cortex (rdACC), and Amy_L between the Alex and the HC groups (Table 3, Figure 1).

\section{Comparison analysis of gray matter density between two groups}

The results revealed no significant difference in GM density between the Alex and the HC groups (Table 4).

Table 3 Brain regions in the emotion network showing significant differences on ALFF between the Alex and the HC groups

\begin{tabular}{|c|c|c|c|c|}
\hline & \multicolumn{2}{|l|}{ ALFF value } & \multirow[t]{2}{*}{$t$-value } & \multirow[t]{2}{*}{$p$-value } \\
\hline & Alex group & HC group & & \\
\hline $\mathrm{V} 8 / \mathrm{sCB}, \mathrm{Bi}$ & $0.996 \pm 0.015$ & $0.991 \pm 0.021$ & 0.832 & 0.410 \\
\hline $\mathrm{MT}+, \mathrm{Bi}$ & $1.001 \pm 0.016$ & $1.000 \pm 0.040$ & 0.025 & 0.980 \\
\hline $\mathrm{V} 4, \mathrm{R}$ & $0.886 \pm 0.014$ & $0.893 \pm 0.012$ & -1.574 & 0.123 \\
\hline latOCC/Temp, $\mathrm{R}$ & $0.992 \pm 0.017$ & $0.997 \pm 0.029$ & -0.703 & 0.486 \\
\hline PCC & $1.003 \pm 0.017$ & $0.993 \pm 0.033$ & 1.189 & 0.242 \\
\hline VI* & $1.001 \pm 0.019$ & $1.019 \pm 0.028$ & -2.477 & 0.018 \\
\hline dmPFC & $1.004 \pm 0.019$ & $0.999 \pm 0.022$ & 0.744 & $0.46 \mathrm{I}$ \\
\hline pgACC & $0.993 \pm 0.027$ & $0.995 \pm 0.031$ & -0.254 & 0.801 \\
\hline rdACC* & $1.002 \pm 0.022$ & $0.984 \pm 0.022$ & 2.587 & 0.013 \\
\hline valns/frOP/TP, RpOFC, Bi & $0.998 \pm 0.010$ & $0.989 \pm 0.019$ & 1.784 & 0.082 \\
\hline alns, pOFC, $\mathrm{Bi}$ & $0.998 \pm 0.015$ & $0.997 \pm 0.022$ & 0.125 & 0.901 \\
\hline valns/TC/OFC, Bi & $0.990 \pm 0.015$ & $0.994 \pm 0.021$ & -0.769 & 0.446 \\
\hline midlns, Bi dPut, R & $0.993 \pm 0.024$ & $0.993 \pm 0.023$ & 0.073 & 0.942 \\
\hline vStr, midlns, HCMP, L & $0.995 \pm 0.012$ & $1.001 \pm 0.021$ & -1.048 & 0.301 \\
\hline Amy_L* & $0.989 \pm 0.019$ & $1.002 \pm 0.023$ & $-2.14 \mid$ & 0.038 \\
\hline $\mathrm{Hy}$ & $0.990 \pm 0.020$ & $0.999 \pm 0.019$ & -1.498 & 0.142 \\
\hline PAG/Thal & $0.993 \pm 0.023$ & $1.001 \pm 0.017$ & -1.321 & 0.194 \\
\hline Amy vStr vGP Thal, Bi & $0.995 \pm 0.015$ & $1.000 \pm 0.014$ & -1.065 & 0.293 \\
\hline frOP, $R$ & $1.004 \pm 0.014$ & $0.993 \pm 0.026$ & 1.702 & 0.097 \\
\hline IFG, R & $1.004 \pm 0.011$ & $0.996 \pm 0.021$ & $1.45 \mathrm{I}$ & 0.155 \\
\hline preSMA, IFG, L & $1.004 \pm 0.012$ & $1.004 \pm 0.010$ & 0.000 & 1.000 \\
\hline
\end{tabular}

Notes: Data are presented as percentage/ratio or mean $\pm \mathrm{SD} ;{ }^{*} p<0.05$.

Abbreviations: Alex, alexithymia; alns, POFC, anterior insula, posterior orbitofrontal cortex; ALFF, amplitude of low-frequency fluctuation; Amy vStr vGP Thal, Bi, Bilateral amygdala, ventral striatum, ventral globus pallidus, thalamus; Amy_L, Left basolateral amygdala; dmPFC, dorsal medial prefrontal cortex; frOP, R, right frontal operculum; HC, healthy control; $\mathrm{Hy}$, hypothalamus; IFG, R, right inferior frontal gyrus; latOCC/Temp, R, right lateral occipital cortex/temporal lobe; midlns, Bi dPut, R, Bilateral middle insula, right dorsal putamen; MT+, Bi, bilateral middle temporal complex; PAG/thal, periaqueductal gray/thalamus; PCC, posterior cingulate cortex; pgACC, pregenual anterior cingulate cortex; preSMA, IFG, L, left pre-supplementary motor area, inferior frontal gyrus; rdACC, rostral dorsal anterior cingulate cortex; V4, R, right visual area 4; V8/ sCB, Bi, visual area 8/bilateral superior cerebellum; valns/frOP/TP, RpOFC, Bi, ventral anterior insula/frontal operculum/right temporal pole, bilateral posterior orbitofrontal cortex; valns/TC/OFC, Bi, bilateral ventral anterior insula/temporal cortex/orbitofrontal cortex; vStr, midlns, HCMP, L, left ventral striatum, middle insula, hippocampus. 

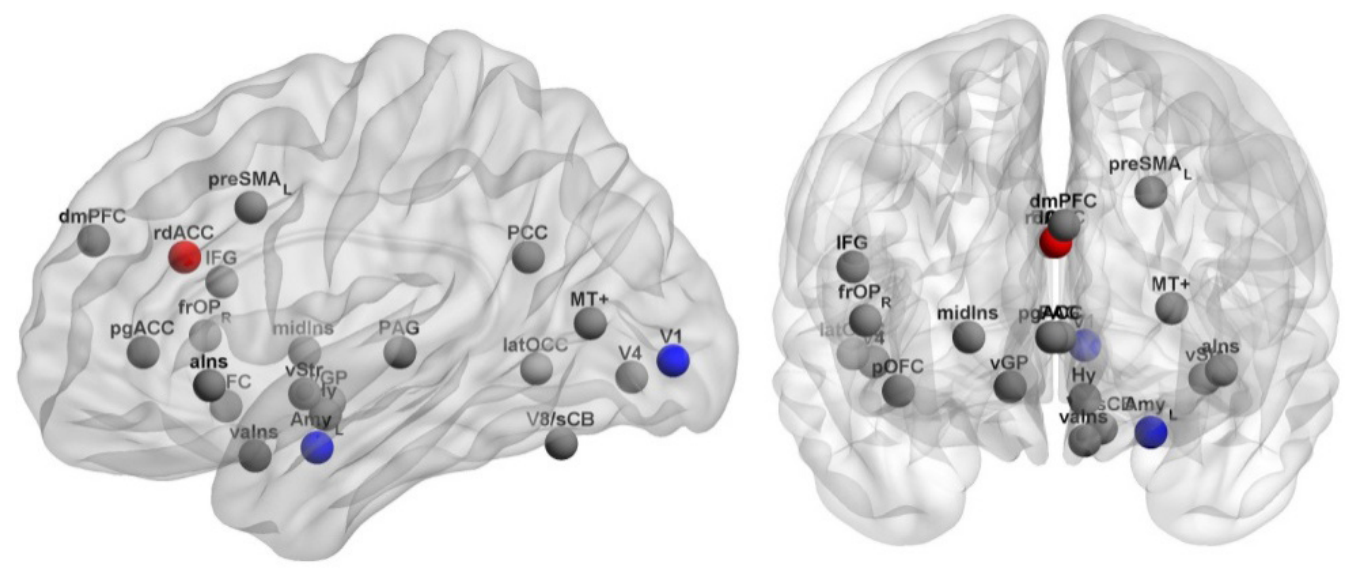

Figure I Brain regions in the emotion network showing significant differences on ALFF between the Alex and the HC groups.

Notes: Red balls indicate Alex group > HC group; blue balls indicate Alex group < HC group; gray balls represent other brain regions in the emotion network.

Abbreviations: Alex, alexithymia; alns, pOFC, anterior insula, posterior orbitofrontal cortex; ALFF, amplitude of low-frequency fluctuation; Amy vStr vGP Thal, Bi, bilateral amygdala, ventral striatum, ventral globus pallidus, thalamus; Amy_L, left basolateral amygdala; dmPFC, dorsal medial prefrontal cortex; frOP, R, right frontal operculum; HC, healthy control; Hy, hypothalamus; IFG, R, right inferior frontal gyrus; latOCC/Temp, R, right lateral occipital cortex/temporal lobe; midlns, Bi dPut, R, bilateral middle insula, right dorsal putamen; MT+, Bi, bilateral middle temporal complex; PAG/thal, periaqueductal gray/thalamus; pgACC, pregenual anterior cingulate cortex; preSMA, IFG, L, left pre-supplementary motor area, inferior frontal gyrus; rdACC, rostral dorsal anterior cingulate cortex; $\mathrm{V} 4, \mathrm{R}$, right visual area 4; V8/sCB, Bi, visual area 8/bilateral superior cerebellum; valns/frOP/TP, RpOFC, Bi, ventral anterior insula/frontal operculum/right temporal pole, bilateral posterior orbitofrontal cortex; valns/TC/OFC, Bi, bilateral ventral anterior insula/temporal cortex/orbitofrontal cortex; vStr, midlns, HCMP, L, left ventral striatum, middle insula, hippocampus.

\section{Comparative analysis of functional and structural connectivity between two groups}

The results revealed weaker FC between Amy_L and Temporal_Sup_L and Amy_L and supplementary motor area (SMA) in the alexithymia group, compared with the HC group (Table 5, Figure 2). The results revealed no significant difference in the FA value between the Alex and the $\mathrm{HC}$ groups (Table 5).

Table 4 Comparison analysis of gray matter on GM density between the Alex and the HC groups

\begin{tabular}{|c|c|c|c|c|}
\hline & \multicolumn{2}{|c|}{ GM density value } & \multirow[t]{2}{*}{ t-value } & \multirow[t]{2}{*}{$p$-value } \\
\hline & Alex group & HC group & & \\
\hline $\mathrm{V} 8 / \mathrm{sCB}, \mathrm{Bi}$ & $0.718 \pm 0.045$ & $0.734 \pm 0.033$ & -1.345 & 0.186 \\
\hline$M T+, B i$ & $0.2298 \pm 0.027$ & $0.235 \pm 0.029$ & -0.602 & $0.55 \mathrm{I}$ \\
\hline $\mathrm{V} 4, \mathrm{R}$ & $0.378 \pm 0.039$ & $0.358 \pm 0.047$ & 1.449 & 0.155 \\
\hline latOCC/Temp, R & $0.438 \pm 0.076$ & $0.446 \pm 0.048$ & -0.429 & 0.670 \\
\hline PCC & $0.651 \pm 0.055$ & $0.668 \pm 0.043$ & -1.107 & 0.275 \\
\hline VI & $0.601 \pm 0.044$ & $0.721 \pm 0.033$ & 0.991 & 0.328 \\
\hline dmPFC & $0.529 \pm 0.048$ & $0.529 \pm 0.039$ & -0.028 & 0.978 \\
\hline PgACC & $0.546 \pm 0.077$ & $0.547 \pm 0.068$ & -0.072 & 0.943 \\
\hline $\mathrm{rdACC}$ & $0.560 \pm 0.095$ & $0.574 \pm 0.064$ & -0.542 & 0.591 \\
\hline valns/frOP/TP, RpOFC, Bi & $0.557 \pm 0.078$ & $0.571 \pm 0.046$ & -0.735 & 0.467 \\
\hline alns, pOFC, $\mathrm{Bi}$ & $0.540 \pm 0.051$ & $0.528 \pm 0.046$ & 0.805 & 0.426 \\
\hline valns/TC/OFC, Bi & $0.43 \mathrm{I} \pm 0.025$ & $0.429 \pm 0.035$ & 0.238 & 0.813 \\
\hline midlns, Bi dPut, R & $0.426 \pm 0.047$ & $0.431 \pm 0.032$ & -0.415 & $0.68 I$ \\
\hline vStr, midlns, HCMP, L & $0.655 \pm 0.039$ & $0.654 \pm 0.042$ & 0.103 & 0.919 \\
\hline Amy_L & $0.700 \pm 0.027$ & $0.706 \pm 0.028$ & -0.663 & 0.511 \\
\hline $\mathrm{Hy}$ & $0.408 \pm 0.025$ & $0.406 \pm 0.028$ & 0.299 & 0.767 \\
\hline PAG/Thal & $0.517 \pm 0.048$ & $0.503 \pm 0.043$ & 1.015 & 0.316 \\
\hline Amy vStr vGP Thal, Bi & $0.348 \pm 0.021$ & $0.345 \pm 0.027$ & 0.499 & 0.621 \\
\hline frOP, $R$ & $0.422 \pm 0.087$ & $0.439 \pm 0.044$ & -0.809 & 0.423 \\
\hline IFG, R & $0.409 \pm 0.075$ & $0.426 \pm 0.036$ & -0.946 & 0.350 \\
\hline preSMA, IFG, L & $0.183 \pm 0.035$ & $0.182 \pm 0.028$ & 0.143 & 0.887 \\
\hline
\end{tabular}

Notes: Data are presented as percentage/ratio or mean $\pm \mathrm{SD}$; there are some significant differences, $p<0.05$.

Abbreviations: Alex, alexithymia; alns, pOFC, anterior insula, posterior orbitofrontal cortex; Amy vStr vGP Thal, Bi, Bilateral amygdala, ventral striatum, ventral globus pallidus, thalamus; Amy_L, left basolateral amygdala; dmPFC, dorsal medial prefrontal cortex; frOP, R, right frontal operculum; GM, gray matter; HC, healthy control; Hy, hypothalamus; IFG, R, right inferior frontal gyrus; latOCC/Temp, R, right lateral occipital cortex/temporal lobe; midlns, Bi dPut, R, Bilateral middle insula, right dorsal putamen; MT+, Bi, bilateral middle temporal complex; PAG/thal, periaqueductal gray/thalamus; pgACC, pregenual anterior cingulate cortex; preSMA, IFG, L, left pre-supplementary motor area, inferior frontal gyrus; rdACC, rostral dorsal anterior cingulate cortex; $\mathrm{V} 4$, R, right visual area 4 ; V8/sCB, Bi, visual area $8 / b i l a t e r a l$ superior cerebellum; valns/ frOP/TP, RpOFC, Bi, ventral anterior insula/frontal operculum/right temporal pole, bilateral posterior orbitofrontal cortex; valns/TC/OFC, Bi, bilateral ventral anterior insula/ temporal cortex/orbitofrontal cortex; vStr, midlns, HCMP, L, left ventral striatum, middle insula, hippocampus. 
Table 5 Comparison of the functional and structural connectivity between the Alex and the HC groups

\begin{tabular}{|c|c|c|c|c|}
\hline & \multicolumn{2}{|l|}{ FC/FA value } & \multirow[t]{2}{*}{ t-value } & \multirow[t]{2}{*}{$p$-value } \\
\hline & HC group & Alex group & & \\
\hline \multicolumn{5}{|l|}{ Functional connectivity (FC value) } \\
\hline VI and Temporal_Sup_L (MNI: $-57,-12,15)^{*}$ & $0.12 \pm 0.041$ & $0.024 \pm 0.01 \mathrm{I}$ & -4.0639 & 0.002 \\
\hline VI and Paracentral_Lobule_L (MNI: $-6,-18,72)^{*}$ & $0.427 \pm 0.075$ & $0.337 \pm 0.065$ & -4.140 & 0.001 \\
\hline Amy_L and Temporal_Sup_L (MNI: $-57,-12,15)^{*}$ & $0.314 \pm 0.047$ & $0.283 \pm 0.044$ & -3.9274 & 0.003 \\
\hline Amy_L and SMA (MNI: I2, -9, 5I)* & $0.105 \pm 0.035$ & $0.038 \pm 0.016$ & -4.771 & $<0.001$ \\
\hline \multicolumn{5}{|l|}{ Structural connectivity (FA value) } \\
\hline VI and Temporal_Sup_L & $0.476 \pm 0.115$ & $0.4 I I \pm 0.174$ & 1.422 & 0.163 \\
\hline VI and Paracentral_Lobule_L & $0.328 \pm 0.214$ & $0.280 \pm 0.226$ & 0.707 & 0.484 \\
\hline Amy_L and Temporal_Sup_L & $0.315 \pm 0.255$ & $0.275 \pm 0.245$ & 0.507 & 0.615 \\
\hline Amy_L and SMA & $0.390 \pm 0.196$ & $0.294 \pm 0.237$ & 1.439 & 0.158 \\
\hline
\end{tabular}

Notes: Data are presented as percentage/ratio or mean $\pm \mathrm{SD} ;{ }^{*} p<0.05$.

Abbreviations: Alex, alexithymia; Amy_L, left basolateral amygdala; HC, healthy control; MNI, Montreal Neurological Institute; Paracentral_Lobule_L, left paracentral lobule; SMA, supplementary motor area; Temporal_Sup_L, left superior temporal gyrus.

\section{Discussion}

Alexithymia is a personality trait characterized by emotional dysfunction. This study examined the emotion network in people with alexithymia. Compared with the HC group, the Alex group displayed higher intrinsic neural activity in rdACC and lower activity in V1 and the Amy_L. The ACC includes two parts: the dorsal ACC (dACC) and the ventral ACC. The dACC is in charge of the detection and appraisal of emotional and social information. ${ }^{30}$ Furthermore, alexithymia is linked to the blunted response of dACC to social rejection. ${ }^{31}$ The rdACC plays a role in regulating the startle reaction and mediating the anxiety emotion..$^{32}$ In the present study, the part of dACC associated with alexithymia is more precisely located in the rdACC. The V1 is in charge of processing visual information and regulating pattern recognition. ${ }^{33}$ It has been reported that people with high-level alexithymia have difficulty in processing visual information from emotional body postures. ${ }^{34}$ Therefore, a low activity in V1 is closely related to alexithymia. The Amy_L belongs to the limbic system and plays a key role in memory and emotional reactions. ${ }^{35}$ Alexithymia is characterized by emotional dysfunction and contributes to social function impairment. ${ }^{36-38}$ A previous study reported that Amy_L was associated with alexithymia. ${ }^{36}$ The present study also supports this finding.

Based on the fact that three brain regions show abnormal intrinsic neural activity in people with alexithymia, the present study further investigated the roles of these brain regions in the occurrence of alexithymia. As a multifaceted personality trait, alexithymia should be associated with the
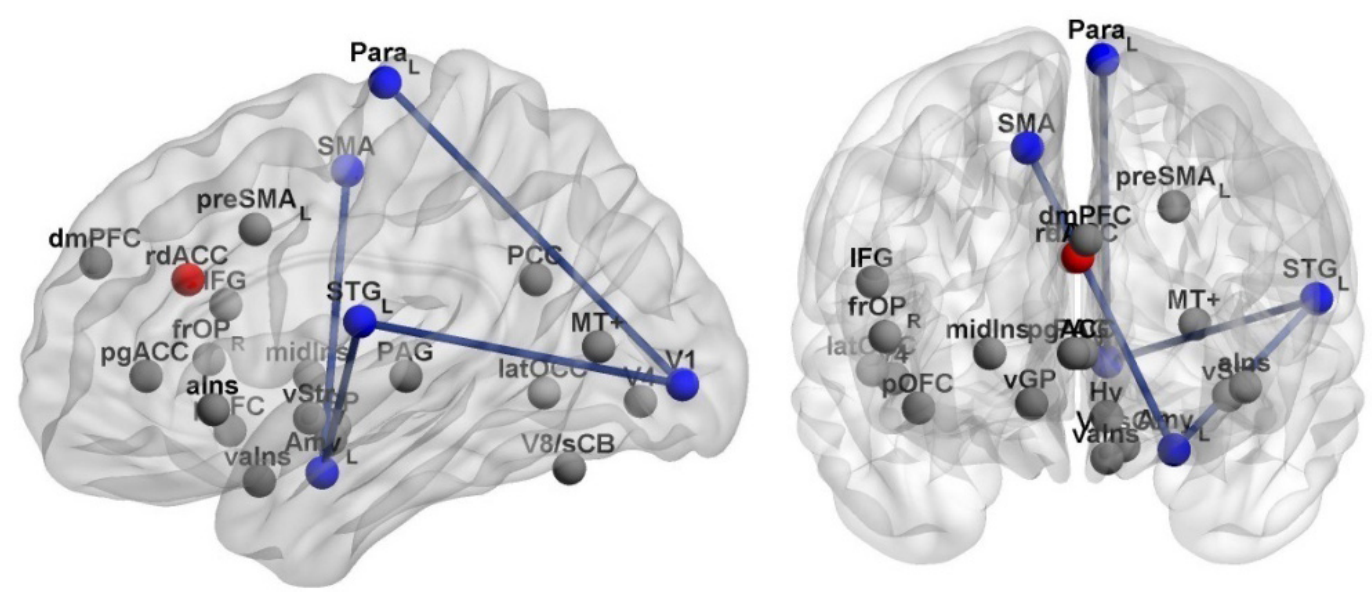

Figure 2 The functional connectivity showing significant differences between the Alex and the HC groups.

Notes: Red balls indicate Alex group $>\mathrm{HC}$ group; blue balls indicate Alex group $<\mathrm{HC}$ group and the brain regions with weak functional connectivity; gray balls represent other brain regions in the emotion network. Blue lines indicate weak functional connectivity in Alex group, compared with HC group.

Abbreviations: Alex, alexithymia; dmPFC, dorsal medial prefrontal cortex; frOP, alns, anterior insula, frontal operculum; HC, healthy control; Hy, hypothalamus; IFG, inferior frontal gyrus; latOCC, lateral occipital cortex; MT+, middle temporal complex; PAG, periaqueductal gray; PCC, posterior cingulate cortex; PARA, paracentral lobule; PgACC, pregenual anterior cingulate cortex; POFC, anterior insula, posterior orbitofrontal cortex; preSMA, L, left pre-supplementary motor area; STG, superior temporal gyrus; V8/sCB, visual area 8/ superior cerebellum; valns, ventral anterior insula; vGP, ventral globus pallidus; vStr, ventral striatum. 
cooperative action of numerous brain regions. This study identifieed weak FC between V1 and Temporal_Sup_L and V1 and Paracentral_Lobule_L in alexithymia subjects. The Temporal_Sup_L is the primary auditory cortex, and it plays a key role in language comprehension. ${ }^{39}$ The Paracentral_ Lobule is the continuation of the precentral and postcentral gyrus and regulates motor and sensory innervations. ${ }^{40,41}$ Identifying and describing one's own feelings (including visual and auditory senses) are involved in language comprehension, motor controls and sensory information processing. Moreover, the present study presented a weak FC between Amy_L and Temporal_Sup_L and Amy_L and SMA in the alexithymia group. The SMA plays a key role in movement control, such as bimanual coordination and postural stability during standing or walking, which are all involved in identifying and describing emotional feelings. ${ }^{42-44}$ Therefore, the result of this study implies that the difficulties in identifying emotion and expression (the core symptoms of alexithymia) could be associated with weak FC between V1 and Temporal_Sup_L; V1 and Paracentral_Lobule_L; Amy_L and Temporal_Sup_L; and Amy_L and SMA. In the present study, no abnormal functional connection was identified between rdACC and other brain regions. This indicates that the dysfunction of rdACC alone is involved in the occurrence of alexithymia, and thus rdACC itself should be a therapeutic target for alexithymia. However, in terms of the V1 and left amygdale, the FC between V1 and Temporal_Sup_L; V1 and Paracentral_Lobule_L; Amy_L and Temporal_Sup_L; and Amy_L and SMA should be considered a therapeutic target.

According to the VBM and DTI analysis results, it is notable that no significant difference was identified in the gray matter or structural connectivity in the emotion networks of people with and without alexithymia. This suggests that the occurrence of alexithymia might only be due to a slight alteration in the neural activity of $\mathrm{V} 1, \mathrm{rdACC}$, and left amygdala, and that it is not associated with any structural brain alteration. This is why alexithymia is considered just a personality trait rather than an emotional disorder. Most emotional disorders are caused not only by neural activity alteration but also by structural alteration. Furthermore, it implies that some noninvasive treatment technologies focusing on improving neural activity (such as transcranial magnetic stimulation, psychotherapy, and physical therapy) may be effective in helping people with alexithymia.

In conclusion, this study focused on the functional and structural characteristics of the emotion network in people with alexithymia. There were several limitations to this study. The emotion network's functional characteristics should be investigated during emotionally motivated situations. Moreover, depression and anxiety, which are closely related to alexithymia, should be measured and analyzed. This study identified the altered intrinsic neural activity in V1, rdACC, and the left amygdala, as well as the weak functional connectivity between V1 and Temporal_Sup_L; V1 and Paracentral_Lobule_L; Amy_L and Temporal_Sup_L; and Amy_L and SMA in people with alexithymia. This study did not identify any structural alteration of the emotion network in people with alexithymia. However, the results of this study can help identify treatment options and may have heuristic value. To further investigate the features of the brain regions in people with alexithymia, a series of studies using different neural imaging methods is necessary.

\section{Acknowledgments}

This work was supported by a grant from Zhejiang Provincial Natural Science Foundation of China (No LQ14H090004) and the China Scholarship Council (201708330165).

\section{Author contributions}

Han Dai designed the study, managed the imaging data selection and analysis, and wrote the first draft of the manuscript. Mei Li, Minjun Mei and Xiaofei Sun undertook the psychology measurement and statistical analysis. All authors contributed toward data analysis, drafting and critically revising the paper, gave final approval of the version to be published, and agree to be accountable for all aspects of the work.

\section{Disclosure}

The authors report no conflicts of interest in this work.

\section{References}

1. Taylor GJ, Bagby RM, Parker JDA. What's in the name "alexithymia"? A commentary on "Affective agnosia: Expansion of the alexithymia construct and a new opportunity to integrate and extend Freud's legacy.' Neurosci Biobehav Rev. 2016;68:1006-1020.

2. Taylor GJ. Recent developments in alexithymia theory and research. Can J Psychiatry. 2000;45(2):134-142

3. Waller E, Scheidt CE. Somatoform disorders as disorders of affect regulation: a development perspective. Int Rev Psychiatry. 2006;18(1):13.

4. Lumley MA, Neely LC, Burger AJ. The assessment of alexithymia in medical settings: implications for understanding and treating health problems. J Pers Assess. 2007;89(3):230-246.

5. Shah $P$, Hall R, Catmur C, Bird G. Alexithymia, not autism, is associated with impaired interoception. Cortex. 2016;81:215-220.

6. Ghiggia A, Romeo A, Tesio V, et al. Alexithymia and depression in patients with fibromyalgia: when the whole is greater than the sum of its parts. Psychiatry Res. 2017;255:195-197.

7. Tella MD, Enrici I, Castelli L, et al. Alexithymia, not fibromyalgia, predicts the attribution of pain to anger-related facial expressions J Affect Disord. 2017;227:272-279. 
8. Kober H, Barrett LF, Joseph J, Blissmoreau E, Lindquist K, Wager TD. Functional grouping and cortical-subcortical interactions in emotion: a metaanalysis of neuroimaging studies. Neuroimage. 2008;42(2):998-1031.

9. Phillips ML, Drevets WC, Rauch SL, Lane R. Neurobiology of emotion perception II: Implications for major psychiatric disorders. Biol Psychiatry. 2003;54(5):515-528.

10. Champagne J, Lakis N, Bourque J, Stip E, Lipp O, Mendrek A. Progesterone and cerebral function during emotion processing in men and women with schizophrenia. Schizophr Res Treatment. 2012;2012:917901.

11. Ursu S, Kring AM, Gard MG, et al. Prefrontal cortical deficits and impaired cognition-emotion interactions in schizophrenia. Am J Psychiatry. 2011;168(3):276-285.

12. Sass K, Habel U, Kellermann T, Mathiak K, Gauggel S, Kircher T. The influence of positive and negative emotional associations on semantic processing in depression: an fMRI study. Hum Brain Mapp. 2014; 35(2):471-482.

13. Spielberg JM, Miller GA, Warren SL, Sutton BP, Banich M, Heller W. Transdiagnostic dimensions of anxiety and depression moderate motivation-related brain networks during goal maintenance. Depress and Anxiety. 2014;31(10):805-813.

14. Yuan H, Young KD, Phillips R, Zotev V, Misaki M, Bodurka J. Restingstate functional connectivity modulation and sustained changes after real-time functional magnetic resonance imaging neurofeedback training in depression. Brain Connect. 2014;4(9):690-701.

15. Moriguchi Y, Komaki G. Neuroimaging studies of alexithymia: physical, affective, and social perspectives. Biopsychosoc Med. 2013; $7(1): 1-12$.

16. Goerlichdobre KS, Bruce L, Martens S, Aleman A, Hooker CI. Distinct associations of insula and cingulate volume with the cognitive and affective dimensions of alexithymia. Neuropsychologia. 2014; 53(4):284-292.

17. Ihme K, Sacher J, Lichev V, et al. Alexithymic features and the labeling of brief emotional facial expressions - An fMRI study. Neuropsychologia. 2014;64:289-299.

18. Liemburg EJ. Altered resting state connectivity of the default mode network in alexithymia. Soc Cogn Affect Neurosci. 2012;7(6):660-666.

19. Bhadelia RA, Price LL, Tedesco KL, et al. Diffusion tensor imaging, white matter lesions, the corpus callosum and gait in the elderly. Stroke. 2009;40(12):3816-3820.

20. Ho NS, Wong MM, Lee TM. Neural connectivity of alexithymia: specific association with major depressive disorder. J Affect Disord. 2016; 193:362-372.

21. Bagby RM, Parker JD, Taylor GJ. The twenty-item Toronto Alexithymia Scale - I. Item selection and cross-validation of the factor structure. J Psychosom Res. 1994;38(1):23-32.

22. Song XW, Dong ZY, Long XY, et al. REST: a toolkit for resting-state functional magnetic resonance imaging data processing. PLoS One. 2011;6(9):e25031.

23. Yan CG, Zang YF. DPARSF: a MATLAB toolbox for "pipeline" data analysis of resting-state fMRI. Front Syst Neurosci. 2010;4(13):13.

24. Ran Q, Chen J, Li C, et al. Abnormal amplitude of low-frequency fluctuations associated with rapid-eye movement in chronic primary insomnia patients. Oncotarget. 2017;8(49):84877-84888.

25. Liang P, Deshpande G, Zhao S, Liu J, Hu X, Li K. Altered directional connectivity between emotion network and motor network in Parkinson's disease with depression. Medicine. 2016;95(30):e4222.
26. Kuhn S, Gallinat J. Brains online: structural and functional correlates of habitual Internet use. Addict Biol. 2015;20(2):415-422.

27. Shi Y, Zeng Y, Wu L, et al. A study of the brain abnormalities of poststroke depression in frontal lobe lesion. Sci Rep. 2017;7(1):13203.

28. Xia M, Wang J, Yong H. BrainNet Viewer: a network visualization tool for human brain connectomics. PLoS One. 2013;8(7):e68910.

29. Jiang H, van Zijl PC, Kim J, Pearlson GD, Mori S. DTIStudio: resource program for diffusion tensor computation and fiber bundle tracking. Comput Methods Programs Biomed. 2006;81(2):106-116.

30. Katarina D, Slavich GM, Muscatell KA, Irwin MR, Eisenberger NI. Dorsal anterior cingulate cortex responses to repeated social evaluative feedback in young women with and without a history of depression. Front Behav Neurosci. 2016;10(261):64.

31. Martínezvelázquez ES, Ramosloyo J, Gonzálezgarrido AA, Sequeira H. Feedback-related negativity is enhanced in adolescence during a gambling task with and without probabilistic reinforcement learning. Neuroreport. 2015;26(2):45.

32. Wager TD, van Ast VA, Hughes BL, Davidson ML, Lindquist MA, Ochsner KN. Brain mediators of cardiovascular responses to social threat, part II: prefrontal-subcortical pathways and relationship with anxiety. Neuroimage. 2009;47(3):836-851.

33. Lindeberg T. A computational theory of visual receptive fields. Biol Cybern. 2013;107(6):589-635.

34. Borhani K, Borgomaneri S, Làdavas E, Bertini C. The effect of alexithymia on early visual processing of emotional body postures. Biol Psychol. 2016;115:1-8.

35. Amunts K, Kedo O, Kindler M, et al. Cytoarchitectonic mapping of the human amygdala, hippocampal region and entorhinal cortex: intersubject variability and probability maps. Anat Embryol. 2005;210(5-6):343-352.

36. Goerlich KS, Votinov M, Lammertz SE, et al. Effects of alexithymia and empathy on the neural processing of social and monetary rewards. Brain Struct Func. 2016;222(5):2235-2250.

37. Panayiotou G, Constantinou E. Emotion dysregulation in alexithymia: startle reactivity to fearful affective imagery and its relation to heart rate variability. Psychophysiology. 2017;54(9):1323-1334.

38. Silva AND, Vasco AB, Watson JC. Alexithymia and emotional processing: a mediation model. J Clin Psychol. 2016;73(9):1196-1205.

39. Vander GM, Bourguignon M, Op DBM, et al. Left superior temporal gyrus is coupled to attended speech in a cocktail-party auditory scene. J Neurosci. 2016;36(5):1596-1606.

40. Imamura $\mathrm{T}$, Tsuburaya $\mathrm{K}$. Absence of neurobehavioral disturbance in a focal lesion of the left paracentral lobule. Behav Neurol. 1992; 5(3):189-191.

41. Spasojević G, Malobabic S, Pilipovićspasojević O, Djukićmacut N, Maliković A. Morphology and digitally aided morphometry of the human paracentral lobule. Folia Morphol. 2013;72(1):10-16.

42. Brinkman C. Lesions in supplementary motor area interfere with a monkey's performance of a bimanual coordination task. Neurosci Lett. 1981;27(3):267-270.

43. Penfield $\mathrm{W}$, Welch $\mathrm{K}$. The supplementary motor area of the cerebral cortex; a clinical and experimental study. AMA Arch Neurol Psychiatry. 1951;66(3):289.

44. Serrien DJ, Strens LH, Oliviero A, Brown P. Repetitive transcranial magnetic stimulation of the supplementary motor area (SMA) degrades bimanual movement control in humans. Neurosci Lett. 2002; 328(2):89-92.

\section{Publish your work in this journal}

Neuropsychiatric Disease and Treatment is an international, peerreviewed journal of clinical therapeutics and pharmacology focusing on concise rapid reporting of clinical or pre-clinical studies on a range of neuropsychiatric and neurological disorders. This journal is indexed on PubMed Central, the 'PsycINFO' database and CAS,

\section{Dovepress}

and is the official journal of The International Neuropsychiatric Association (INA). The manuscript management system is completely online and includes a very quick and fair peer-review system, which is all easy to use. Visit http://www.dovepress.com/testimonials.php to read real quotes from published authors. 